\title{
The CMUnited-99 Small-Size Robot Team
}

\author{
Manuela Veloso, Michael Bowling, and Sorin Achim \\ School of Computer Science \\ Carnegie Mellon University \\ Pittsburgh, PA 15213-3891 \\ $\{\mathrm{mmv}, \mathrm{mhb}$, sorin $\}$ ccs.cmu.edu
}

One of the necessary steps in entering a small-size RoboCup team is the actual construction of the robots. We have successfully built robots for RoboCup97 and RoboCup-98, leading to two champion teams, namely CMUnited-97 [2] and CMUnited-98 [1].

Given that our research is focused on team work in multirobot systems and not particularly on the mechanical construction of robots, and given that we had already built two different teams of robots, we decided that we would use the CMUnited-98 robots for the RoboCup-99 competition. We still had to rebuild the goalie robot, as our RoboCup-98 goalie was slightly beyond the size of the allowed robots, according to the revised rules for RoboCup-99.

Our purpose therefore entering RoboCup-99 was to concentrate our effort in new research directions involving more elaborate robot motion and strategic team work, namely:

- Extension of the motion control algorithm to allow for a preferred side, so that attackers would make more and better use of the kicking device.

- Increase of the number of defenders to two from the single one used in CMUnited-98. The number of defenders would vary dynamically in response to the number of attackers.

- Improvement of the coordination between the goalie and the defenders, to maximize the coverage of the defense area and not endanger our own goal.

- Additional strategic planning to dynamically adjust the number of attackers and defenders based on the current situation (e.g. position of the ball, the "formation" of the opponent, or the current score).

We partially successfully pursued our research. We created a sophisticated simulator where we developed an interesting algorithm for the coordination of the goalkeeper and multiple defenders. We expect to use this algorithm in our future RoboCup teams and refine it then, when effectively tested on real robots.

We had however limited success carrying ahead our new approaches in the robot team, as we encountered several unexpected difficulties with the radio control of the five robots. We also realized earlier, but mainly at the competition, that our robots' hardware was indeed worn out, most probably from the several trips and its extensive usage for two years.

We look forward to participating in future RoboCup competitions necessarily with new robots! 
We thank Kwun Han for the development of the vision algorithm of CMUnited98, which we also used in CMUnited-99. We also thank Peter Stone, Rune Jensen, Jim Bruce, and Tucker Balch for their help during the competition.

\section{References}

1. Manuela Veloso, Michael Bowling, Sorin Achim, Kwun Han, and Peter Stone. The CMUnited-98 champion small robot team. In Minoru Asada and Hiroaki Kitano, editors, RoboCup-98: Robot Soccer World Cup II, pgs. 61-76, Springer, 1999.

2. Manuela Veloso, Peter Stone, Kwun Han, and Sorin Achim. The CMUnited-97 small robot team. In Hiroaki Kitano, editors, Robo Cup-97: Robot Soccer World Cup I, pgs. 242-256, Springer, 1998. 